\title{
Intramuscular Clodronate in Long-Term Treatment of Symptomatic Knee Osteoarthritis: A Randomized Controlled Study
}

\author{
Bruno Frediani ${ }^{1} \cdot$ Carmela Toscano $^{1} \cdot$ Paolo Falsetti $^{1} \cdot$ Antonella Nicosia $^{1} \cdot$ Serena Pierguidi ${ }^{1} \cdot$ Alberto Migliore $^{2}$. \\ Stefano Giannotti ${ }^{3} \cdot$ Luca Cantarini $^{1} \cdot$ Edoardo Conticini $^{1}$
}

Published online: 20 February 2020

(c) The Author(s) 2020

\begin{abstract}
Background and Objective Clodronate is a nitrogen-free bisphosphonate that is widely and effectively used in the treatment of many osteo-metabolic disorders. The objective of our study was to evaluate the effectiveness of clodronate in reducing pain and bone marrow edema in knee osteoarthritis.

Methods In total, 74 patients were included in the study. Group 1 received intramuscular clodronate $200 \mathrm{mg}$ daily for 15 days and then once weekly for the next 11.5 months; group 2 received intramuscular clodronate $200 \mathrm{mg}$ daily for 15 days and then once weekly for the next 2.5 months. Visual analog scale (VAS) scores were recorded at baseline (T0) and after 30 days (T1), 3 months (T2), 6 months (T3), 9 months (T4), and 12 months (end of study; T5). We also evaluated functional status and use of paracetamol (T0, T1, T2, T3, T4, and T5) and changes in Whole Organ Magnetic Resonance Imaging Score (WORMS; T0, T2, and T5).

Results Both groups had a statistically significant reduction in VAS score until 3 months. Group 1 then experienced further VAS reductions, whereas VAS scores for group 2 progressively increased. Pain, stiffness, and physical function also showed the same trend, as did bone marrow edema extension, which was evaluated with WORMS.

Conclusion Our study indicates that intramuscular administration of a therapeutic dose of clodronate followed by a maintenance dose is effective in the management of symptomatic knee osteoarthritis, improving functional outcomes and reducing pain and bone marrow edema. Prolonged treatment increases the long-term efficacy of clodronate compared with the shorter schedule.
\end{abstract}

\section{Key Points}

Intramuscular clodronate is well-tolerated and effective for the management of symptomatic knee osteoarthritis.

The efficacy of clodronate is directly proportional to the treatment duration.
Bruno Frediani

fredianibruno60@gmail.com

Institute of Rheumatology, University of Siena, Siena, Italy

2 Unit of Rheumatology, San Pietro Fatebenefratelli Hospital, Rome, Italy

3 Orthopedics Unit, University of Siena, Siena, Italy

\section{Introduction}

Osteoarthritis (OA) is highly prevalent worldwide and usually causes severe pain and disability [1]. Since the early pathogenesis of the disease remains unknown, no drugs are yet available to stop the progression of OA. However, it is important that treatments to control pain and improve both physical activity levels and quality of life for patients are developed.

The pathogenesis of pain in OA is also uncertain, but the extensive use of magnetic resonance imaging (MRI) over recent decades has enabled researchers to understand some important points. The radiological detection of bone marrow edema (BME) in symptomatic joints and its correlation with OA development was an important step in understanding the progression of the disease [2-4].

Since an altered signal pattern seems related to lymphocytic infiltrates, fibrosis, increased vascularization, less 
mineralized bone, and real local edema, the preferred term in most recent literature OA is bone marrow lesions (BML).

However, more recent studies found some important correlations between BML and progression of pain and OA. Indeed, all histopathological studies have involved patients with severe joint disease, in whom the only therapeutic option was a prosthesis [4-7].

In recent years, bisphosphonates have proven their efficacy in the treatment of some diseases with a finding of BML and BME, especially at high dosages and with parenteral administration. However, continuous administration of bisphosphonates, even at lower dosages, has proven to have symptomatic effectiveness [8-11].

Clodronate is a nitrogen-free bisphosphonate that is widely and effectively used in the treatment of many osteometabolic disorders [12-16]. In particular, we highlight its efficacy in the treatment of algodystrophic syndrome [17], hand-erosive OA [18], and knee OA (intra-articular clodronate) [19].

The aim of our study was to evaluate the efficacy of two therapeutic regimens of intramuscular clodronate, using identical initial therapeutic doses but different maintenance doses. The initial therapeutic dose was the same as used by Varenna et al. [17] (intravenous clodronate $300 \mathrm{mg}$ daily for 10 days) for the treatment of algodystrophy.

The safety of our proposed regimen was evidenced by the overall low incidence of adverse events during clodronate treatment [20] and by the safety reported by Frediani et al. [21], who used intramuscular clodronate $100 \mathrm{mg}$ once or twice weekly in the treatment of postmenopausal osteoporosis.

\section{Materials and Methods}

We included all patients who attended the Rheumatology Department of Policlinico Le Scotte, Siena, Italy, for knee pain from January 2013 to December 2014 who were aged $>40$ years and met the following inclusion criteria: knee OA according to American College of Rheumatology criteria [22], Kellgren-Lawrence radiographic score $\geq 2$ in the tibiofemoral joint [23], worsening chronic knee pain lasting from 2 weeks to 43 months, visual analogue scale $($ VAS) $>40 / 100 \mathrm{~mm}$, BML diameter $>0.5 \mathrm{~cm}$ as seen in a knee MRI, and lumbar or femoral T-score less than -2.5 .

The main outcome of the study was variations in pain intensity using a VAS score. VAS score was recorded at baseline (T0) and after 30 days (T1), 3 months (T2), 6 months (T3), 9 months (T4), and 12 months (end of study; T5). As secondary endpoints, we evaluated functional status (Western Ontario and McMaster University Osteoarthritis Index [WOMAC] questionnaire-pain, stiffness and physical function-at T0, T1, T2, T3, T4, and T5), patient use of paracetamol (T0, T1, T2, T3, T4, and T5), and Whole Organ Magnetic Resonance Imaging Score (WORMS) modifications (T0, T2, and T5).

The exclusion criteria were hypocalcemia; hypercalcemia; low glomerular filtration rate $(<30 \mathrm{~mL} / \mathrm{min})$; liver, kidney, heart, lung, or neurological diseases; diabetes; tumors; previous treatment with bisphosphonates; and knee pain due to trauma.

Intra-articular injection of steroids and/or hyaluronic acid was not permitted during the study or in the 3 months before.

During the study, patients were not permitted to use nonsteroidal anti-inflammatory drugs but were allowed to take paracetamol up to $3 \mathrm{~g}$ daily and were required to record consumption in their own diary. Paracetamol consumption was expressed in average grams per patient per day.

All procedures that involved human participants were in accordance with the ethical standards of the national and Hospital of Siena research committee and with the 1964 Helsinki declaration and its later amendments or comparable ethical standards. All patients provided written consent to participate in the study.

\subsection{Randomization}

Patients were randomized into two parallel treatment groups: Group 1 received intramuscular clodronate $200 \mathrm{mg}$ daily for 15 days and then once weekly for the next 11.5 months; group 2 received intramuscular clodronate $200 \mathrm{mg}$ daily for 15 days and then once weekly for the next 2.5 months.

\subsection{Knee Magnetic Resonance Imaging}

Each patient underwent 3-D high-resolution MRI at T0, T2, and T5. Every MRI was evaluated by an expert musculoskeletal radiologist blinded to patient grouping. The images were acquired in coronal, axial, and sagittal planes, with a field of view of $18 \mathrm{~cm}$ and $256 \times 256$ matrix, with an $0.5 \mathrm{~mm}$ intersection gap.

T1-weighted spin-echo MRI scans were acquired in coronal, axial, and sagittal planes (repetition time [TR] $580 \mathrm{~ms}$, echo time [TE] $12 \mathrm{~ms}$ ); T2-weighted spin-echo scans were acquired in axial and sagittal planes (TR $4000 \mathrm{~ms}$, TE 30/100 ms); proton-density fat-suppression-weighted scans were acquired in a coronal plane (TR $2800 \mathrm{~ms}$, TE $40 \mathrm{~ms}$ ).

Subchondral BMLs were identified as high-intensity signal areas on T2-weighted fat-suppression imaging and analyzed using WORMS [24].

BMLs were codified from 0 to 3 in each of the ten subregions of the medial and lateral tibiofemoral compartment and in each of the four subregions of the patellofemoral compartment by an experienced radiologist. The extent of lesions was evaluated using the WORMS scale $(0=$ no edema; $1=<25 \% ; 2=25-50 \% ; 3=>50 \%)$, and a total 
WORMS was calculated by adding the score for each subregion together.

\subsection{Statistical Analysis}

We computed the statistical significance using a two-tailed test, with a significance level set at $<0.05$.

According to the results of previous studies of pain reduction in patients treated with zoledronate [25] and neridronate [26], we anticipated a VAS score reduction of $>20 \mathrm{~mm}$ in patients treated with clodronate.

We analyzed results according to the "intention-to-treat" principle, including all randomized patients who received at least one dose of clodronate. Since our outcomes were not normally distributed (Shapiro-Wilk test), we used nonparametric tests.

We used the Mann-Whitney $U$ test to evaluate differences between the two groups and the Wilcoxon-signed rank test to evaluate the intra-group differences and WORMS score at $\mathrm{T} 1, \mathrm{~T} 2, \mathrm{~T} 3, \mathrm{~T} 4$, and $\mathrm{T} 5$.

Predictive factors for VAS and WORMS modifications were evaluated using univariate and multivariate regression analysis.

We analyzed both absolute and percentage differences. All statistical tests were bilateral, with $p<0.05$, and evaluated using SPSS software (version 17.0; IBM, Armonk, NY, USA).

\section{Results}

A total of 74 patients were included in the study, equally distributed between the two groups. No patient withdrew before the end of the study. The two groups were similar at T0 (Table 1).

For both groups, VAS statistically significantly reduced until 3 months (T2), from $62.4 \pm 13.6$ (standard deviation) to $10.3 \pm 8.9$ in group 1 and from $68.7 \pm 17.3$ to $11.2 \pm 9.4$ in group 2. Group 1 then experienced a further VAS reduction at T3 (9.3 \pm 7.8$)$, with minimal changes at T4 (12.4 \pm 8.7$)$ and T5 (15.6 \pm 9.8$)$, whereas VAS scores for group 2 progressively increased $(28.8 \pm 20.3$ at $\mathrm{T} 3 ; 43.8 \pm 30.1$ at $\mathrm{T} 4$; $50.3 \pm 31.9$ at T5) (Fig. 1).

The same trend was also observed for WOMAC score (pain, stiffness, physical function) (Table 2).

The consumption of paracetamol decreased in both groups until T2 (group 1: $1.16 \pm 0.6$ at T0; $0.72 \pm 0.5$ at $\mathrm{T} 1 ; 0.55 \pm 0.5$ at $\mathrm{T} 2$; group $2: 1.13 \pm 0.6$ at $\mathrm{T} 0 ; 0.72 \pm 0.4$ at $\mathrm{T} 1 ; 0.64 \pm 0.4$ at $\mathrm{T} 2$ ). The average intake of paracetamol increased in group $2(0.94 \pm 0.6$ at $\mathrm{T} 3 ; 1.04 \pm 0.7$ at $\mathrm{T} 4$; $1.05 \pm 0.7$ at T5) but further reduced in group 1 at T3 and $\mathrm{T} 4(0.42 \pm 0.4$ and $0.28 \pm 0.2$, respectively), remaining substantially stable at T5 $(0.29 \pm 0.2)$.
Table 1 Patient characteristics at baseline (T0)

\begin{tabular}{llll}
\hline Characteristics & Group 1 $(n=37)$ & Group 2 $(n=37)$ & $p$ value \\
\hline Age (years) & $68.3 \pm 13.7$ & $66.1 \pm 12.1$ & 0.2 \\
Sex (F/M), $n$ & $24 / 13$ & $20 / 17$ & 0.5 \\
BMI, kg/m ${ }^{2}$ & 26.3 & 26.8 & 0.8 \\
$\begin{array}{l}\text { Duration of pain, } \\
\quad \text { weeks }\end{array}$ & $12.7 \pm 4.2$ & $11.1 \pm 3.5$ & 0.1 \\
$\begin{array}{l}\text { VAS score (0-100), } \\
\quad \text { mm }\end{array}$ & $62.4 \pm 13.6$ & $68.7 \pm 17.3$ & 0.2 \\
$\begin{array}{l}\text { WOMAC score (pain) } \\
\text { WOMAC score (dis- }\end{array}$ & $6.6 \pm 2.0$ & $7.2 \pm 2.4$ & 0.1 \\
$\quad 20.6 \pm 3.8$ & $22.1 \pm 4.3$ & 0.3 \\
$\quad \begin{array}{l}\text { WOMility) } \\
\quad \text { ness) }\end{array}$ & & $2.9 \pm 1.9$ & 0.9 \\
WORMS & $6.9 \pm 2.6$ & $7.6 \pm 3.7$ & 0.2 \\
\hline
\end{tabular}

Data are presented as mean \pm standard deviation or $N(\%)$ unless otherwise indicated. Group 1: intramuscular clodronate $200 \mathrm{mg}$ daily for 15 days, then once weekly for the next 11.5 months; group 2: intramuscular clodronate $200 \mathrm{mg}$ daily for 15 days, then once weekly for the next 2.5 months

$B M I$ body mass index, $F$ female, $M$ male, VAS visual analog scale, WOMAC Western Ontario and McMaster University Osteoarthritis Index, WORMS whole organ magnetic resonance imaging score

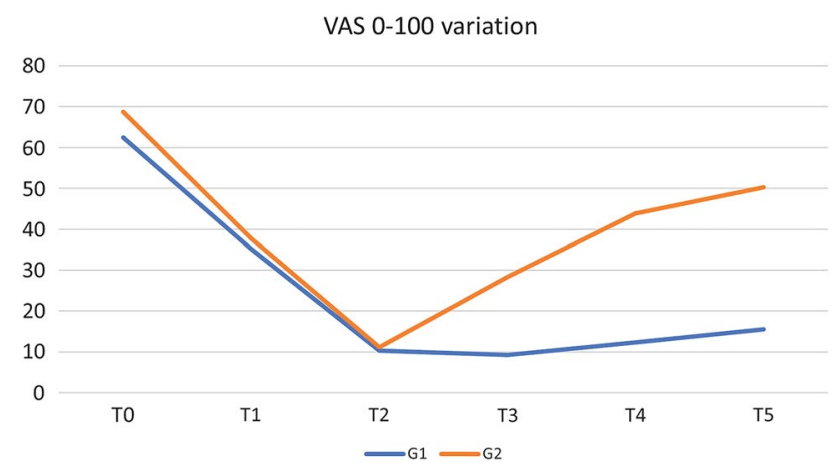

Fig. 1 VAS variation (\%). VAS visual analog scale

BML extension, evaluated by WORMS total score, progressively decreased (group 1: $6.9 \pm 2.6$ at $\mathrm{T} 0$ to $2.1 \pm 1.8$ at T2; group 2: $7.6 \pm 3.7$ at T0 to $2.0 \pm 1.2$ at T2) (Fig. 3); in group 1 , it continued to decrease until T5 $(1.1 \pm 0.8)$, whereas it increased in group 2 (5.9 \pm 4.9 at T5) (Fig. 2).

No adverse events were reported during the study, as expected given the results reported by Frediani et al. [21]. In particular, no difference in serum creatinine or creatinine clearance was found after $3,6,9$, and 12 months of treatment (Fig. 3). 


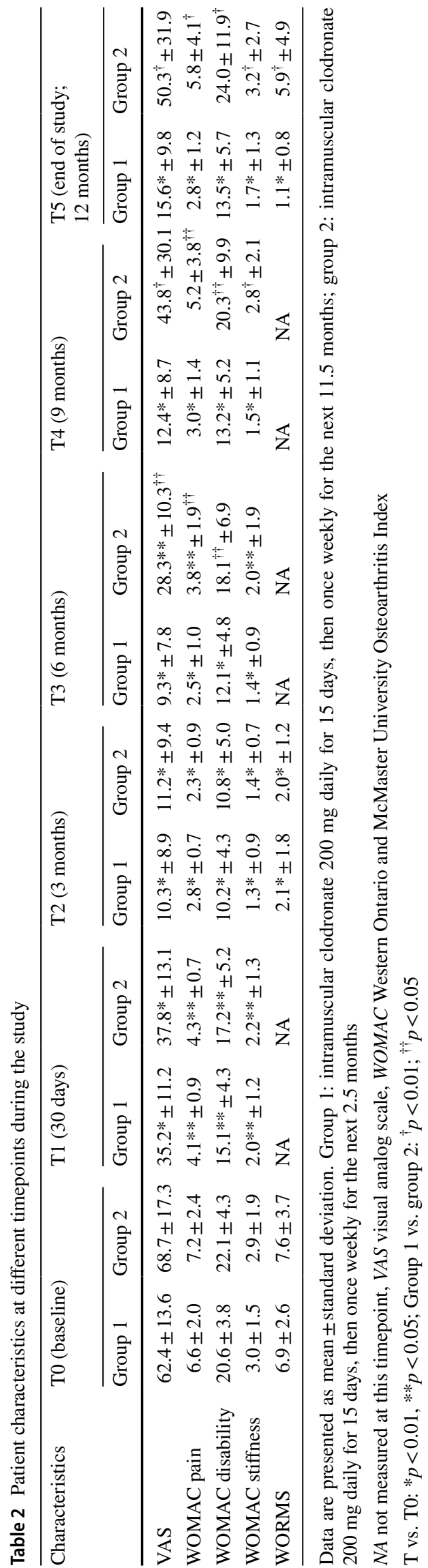

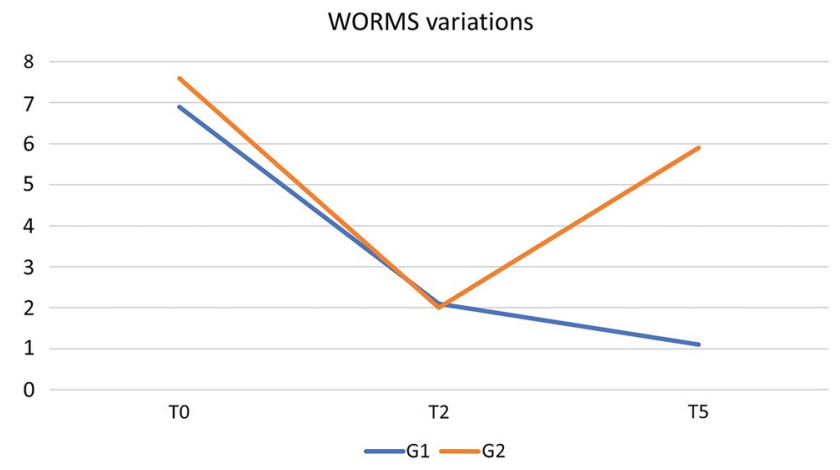

Fig. 2 WORMS variation (\%). WORMS Whole Organ Magnetic Resonance Imaging Score

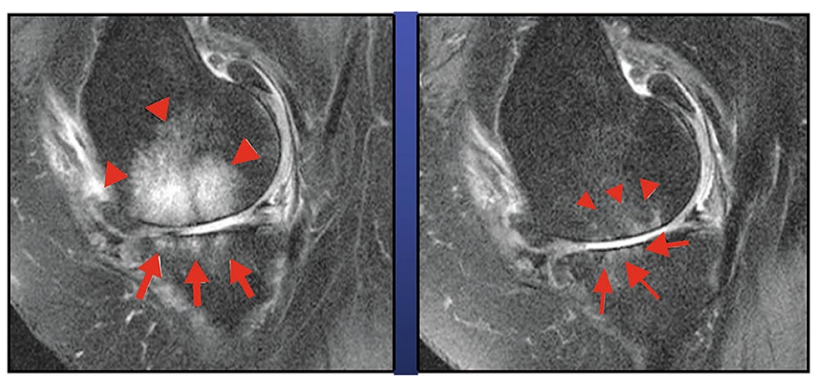

Fig. 3 MRI before and after clodronate therapy (T2 [3 months]). MRI magnetic resonance imaging

\section{Discussion}

Bisphosphonates have proven effective in several conditions characterized by BME and pain, and clodronate in particular is well-known for its anti-inflammatory and analgesic properties $[8,18-20]$ and good safety profile. Intravenous clodronate has proven effective against BME of algodystrophic syndrome when administered at a dose of $300 \mathrm{mg} /$ day for 10 days [17] and has been used to treat painful episodes of erosive OA of the hand [18].

Another case report described the effectiveness of intramuscular clodronate $100 \mathrm{mg}$ in ankle algodystrophic syndrome in a patient with psoriatic arthritis [27].

More powerful molecules, such as amino-bisphosphonates, are widely described in the treatment of algodystrophic syndrome, namely neridronate [28] and alendronate [29]; however, their mechanism of action means they can cause well-known acute phase reactions, especially when administered intravenously.

Only a few papers describe the role of bisphosphonates in $\mathrm{OA}$, with the vast majority investigating clodronate. The role of clodronate in OA, summarized in the recent review by Saviola et al. [30], is an intriguing matter of debate: it may represent a promising therapy even in 
erosive OA [31], in which it was found to be superior over hydroxychloroquine in reducing pain and loss of strength [18]. Moreover, clodronate, the role of which in bone metabolism has been previously summarized and widely described, may also have a role in chondrocyte differentiation, as outlined by Valenti et al. [32], who reported that nanoparticles of clodronate led to SOX9 upregulation. However, none of these studies evaluated the effect of clodronate on BML.

Two previous studies reported that the bisphosphonates neridronate [26] and zoledronate [25] were both effective in terms of reducing pain and physical impairment in knee $\mathrm{OA}$, but neridronate appeared to have a faster onset of action and was better at reducing BML. However, further data on middle- and long-term efficacy are absent.

A strong correlation between BML and pain has been assumed, as pain can be caused by impaired venous drainage, increased intra-osseous pressure, and local acidosis [33].

However, no consensus has been reached on what exactly defines a BML from a histopathologic viewpoint. According to the most recent literature, an altered signal pattern appears to be related to lymphocytic infiltrates, fibrosis, increased vascularization, and less mineralized bone, as well as real local edema [34]; trabecular bone is also involved, and bony remodeling is a common finding [35].

Bisphosphonates are well-known to have both central and peripheral anti-inflammatory and analgesic effects. Interesting applications of bisphosphonates include studies in animal models of collagen-induced arthrosis, where rational use of the antiresorptive drug is described in recent scientific literature emphasizing the role of osteoclastic activation by pro-inflammatory cytokines in the genesis of structural bone damage and erosions. Inhibition of bone resorption, from this viewpoint, is a key point in arthritis therapy [36, 37].

However, in animal models, amino-bisphosphonates exacerbated joint inflammation [38], despite their positive effects on structural bone damage, whereas clodronate had positive effects on inhibition of both structural damage and joint inflammation $[39,40]$.

In particular, Bonabello's studies $[41,42]$ in animal models highlighted a pain-relieving and antinociceptive effect of clodronate at both central and peripheral levels, independent of the antifracture effect. Several reports have highlighted the pain-relieving properties of clodronate in different fields [43-48]. Clodronate's analgesic effect is known and commonly used in clinical practice, including in patients with vertebral fragility fractures [43-45], where the drug had a greater pain-killing effect than other amino-bisphosphonates, including neridronate [46]. In addition, we cannot forget the analgesic role of clodronate in oncology, especially in patients with skeletal involvement $[47,48]$.
Moreover, a recent randomized controlled trial proved the effectiveness of intra-articular clodronate $2 \mathrm{mg}$ per week for 4 weeks in reducing pain compared with $\mathrm{NaCl} 0.9 \%$ [19]; the same working group proved the noninferiority of intraarticular clodronate versus hyaluronic acid [49].

As reported in studies using zoledronate [25] and neridronate [26] in OA, our paper indicates that clodronate reduces pain and BML. Our study is also the first to demonstrate that an initial higher dose of clodronate followed by intramuscular maintenance administration prevented increased BML and pain; patients who stopped treatment did not experience this effect.

Our study has a limitation that should be noted: We used indirect instruments for the evaluation of drug efficacy, such as a pain questionnaire and WORMS. This score measures the extent of edema, but we acknowledge that edema may be related not only to OA but also to many other conditions (trauma, inflammation, and tumor, among others).

\section{Conclusions}

Intramuscular clodronate at a therapeutic dose followed by a maintenance dose is effective in the management of symptomatic knee OA, improving functional outcomes and reducing pain and BML.

Further studies are needed to create a universal treatment schedule for the use of clodronate in this complex disease. In particular, clodronate may offer enhanced protection in early-stage OA, so should be investigated in such patients.

Higher doses of this drug than used in osteoporosis will be necessary, and the duration will need to be longer than that used in algodystrophic syndrome.

Acknowledgements Medical writing was performed by Luca Giacomelli, PhD; editorial assistance was provided by Aashni Shah (Polistudium SRL, Milan, Italy). This assistance was supported by internal funds.

\section{Compliance with Ethical Standards}

Conflicts of Interest Bruno Frediani, Carmela Toscano, Paolo Falsetti, Antonella Nicosia, Serena Pierguidi, Alberto Migliore, Stefano Giannotti, Luca Cantarini and Edoardo Conticini have no conflicts of interest that are directly relevant to the content of this article.

Funding No sources of funding were used to conduct this study or prepare this manuscript.

Ethical Standards All procedures performed in studies involving human participants were in accordance with the ethical standards of the institutional and/or national research committee and with the 1964 Helsinki declaration and its later amendments or comparable ethical standards

Informed Consent Informed consent was obtained from all individual participants included in the study. 
Open Access This article is licensed under a Creative Commons Attribution-NonCommercial 4.0 International License, which permits any non-commercial use, sharing, adaptation, distribution and reproduction in any medium or format, as long as you give appropriate credit to the original author(s) and the source, provide a link to the Creative Commons licence, and indicate if changes were made. The images or other third party material in this article are included in the article's Creative Commons licence, unless indicated otherwise in a credit line to the material. If material is not included in the article's Creative Commons licence and your intended use is not permitted by statutory regulation or exceeds the permitted use, you will need to obtain permission directly from the copyright holder.To view a copy of this licence, visit http://creativecommons.org/licenses/by-nc/4.0/.

\section{References}

1. Lawrence RC, Felson DT, Helmick CG, Arnold LM, Choi H, Deyo RA, et al. Estimates of the prevalence of arthritis and other rheumatic conditions in the United States. Part II. Arthritis Rheum. 2008;58:26-35.

2. Felson DT, Chaisson CE, Hill CL, Totterman SM, Gale ME, Skinner KM, et al. The association of bone marrow lesions with pain in knee osteoarthritis. Ann Intern Med. 2001;134:541-9.

3. Felson DY, McLaughlin S, Goggins J, LaValley MP, Gale ME, Totterman S, et al. Bone marrow edema and its relation to progression of knee osteoarthrtitis. Ann Inter Med. 2003;139:330-6.

4. Zanetti M, Bruder E, Romero J, Hodler J. Bone marrow edema pattern in osteoarthritic knees: correlation between MR imaging and histologic findings. Radiology. 2000;215:835-40.

5. Saadat E, Jobke B, Chu B, Lu Y, Cheng J, Li X, et al. Diagnostic performance of in vivo 3-T MRI for articular cartilage abnormalities in human osteoarthritic knees using histology as standard of reference. Eur Radiol. 2008;18:2292-302.

6. Kazakia GJ, Kuo D, Schooler J, Siddiqui S, Shanbhag S, Bernstein $\mathrm{G}$, et al. Bone and cartilage demonstrate changes localized to bone marrow edemalike lesions within osteoarthritic knees. Osteoarth Cartil. 2013;21:94-101.

7. Yusuf E, Kortekaas MC, Watt I, Huizinga TW, Kloppenburg M. Do knee abnormalities visualised on MRI explain knee pain in knee osteoarthritis? A systematic review. Ann Rheum Dis. 2011;70:60-7.

8. Maksymowych WP, Jhangri GS, Fitzgerald AA, LeClercq S, Chiu P, Yan A, et al. A six-month randomized, controlled, double-blind, dose response comparison of intravenous pamidronate $(60 \mathrm{mg}$ versus $10 \mathrm{mg}$ ) in the treatment of nonsteroidal antiinflammatory drug-refractory ankylosing spondylitis. Arthritis Rheum. 2002;46:766-73.

9. Varenna M, Zucchi F, Binelli L, Failoni S, Gallazzi M, Sinigaglia L. Intravenous pamidronate in the treatment of transient osteoporosis of the hip. Bone. 2002;31:96-101.

10. Tran DQH, Duong S, Bertini P, Finlayson RJ. Treatment of complex regional pain syndrome: a review of the evidence. Can J Anaesth. 2010;57:149-66.

11. Davis AJ, Smith TO, Hing CB, Sofat N. Are bisphosphonates effective in the treatment of osteoarthritis pain? A meta-analysis and systematic review. PLoS One. 2013;8:e72714.

12. Khan SA, McCloskey EV, Nakatsuka K, Orgee J, Coombes GM, Kanis JA. Duration of response with oral clodronate in Paget's disease of bone. Bone. 1996;18(2):185-90.

13. Body JJ, Coleman RE, Piccart M. Use of bisphosphonates in cancer patients. Cancer Treat Rev. 1996;22(4):265-87.

14. Adami S, Bolzicco GP, Rizzo A, Salvagno G, Bertoldo F, Rossini $\mathrm{M}$, et al. The use of dichloromethylene bisphosphonate and aminobutane bisphosphonate in hypercalcemia of malignancy. Bone Miner. 1987;2(5):395-404.

15. Douglas DL, Kanis JA, Paterson AD, Beard DJ, Cameron EC, Watson ME, et al. Drug treatment of primary hyperparathyroidism: use of clodronate disodium. Br Med J. 1983;286(6365):587-90.

16. Shane E, Baquiran DC, Bilezikian JP. Effects of dichloromethylene diphosphonate on serum and urinary calcium in primary hyperparathyroidism. Ann Intern Med. 1981;95(1):23-7.

17. Varenna M, Zucchi F, Ghiringhelli D, Binelli L, Bevilacqua M, Bettica $\mathrm{P}$, et al. Intravenous clodronate in the treatment of reflex sympathetic dystrophy syndrome. A randomized, double blind, placebo controlled study. J Rheumatol 2000;27(6):1477-83.

18. Saviola G, Abdi-Ali L, Campostrini L, Sacco S, Baiardi P, Manfredi $\mathrm{M}$, et al. Clodronate and hydroxychloroquine in erosive osteoarthritis: a 24-month open randomized pilot study. Mod Rheumatol. 2012;22(2):256-63.

19. Rossini M, Adami S, Fracassi E, Viapiana O, Orsolini G, Povino MR, et al. Effects of intra-articular clodronate in the treatment of knee osteoarthritis: results of a double-blind, randomized placebocontrolled trial. Rheumatol Int. 2015;35(2):255-63.

20. Frediani B, Giusti A, Bianchi G, Dalle Carbonare L, Malavolta N, Cantarini L, Saviola G, Molfetta L. Clodronate in the management of different musculoskeletal conditions. Minerva Med. 2018;109(4):300-25.

21. Frediani B, Bertoldi I, Pierguidi S, Nicosia A, Picerno V, Filippou G, Cantarini L, Galeazzi M. Improved efficacy of intramuscular weekly administration of clodronate $200 \mathrm{mg}$ (100 mg twice weekly) compared with $100 \mathrm{mg}$ (once weekly) for increasing bone mineral density in postmenopausal osteoporosis. Clin Drug Investig. 2013;33(3):193-8.

22. Altman R, Asch E, Bloch D, Bole G, Borenstein D, Brandt K et al. Development of criteria for the classification and reporting of osteoarthritis. Classification of osteoarthritis of the knee. Diagnostic and therapeutic criteria committee of the American Rheumatism Association. Arthritis Rheum 1986;29:1039-49.

23. Kellgren JH, Lawrence JS. Radiological assessment of osteoarthrosis. Ann Rheum Dis. 1957;16(4):494-502.

24. Peterfy CG, Guermazi A, Zaim S, Tirman PF, Miaux Y, White $\mathrm{D}$, et al. Whole-Organ Magnetic Resonance Imaging Score (WORMS) of the knee in osteoarthritis. Osteoarth Cartil. 2004;12:177-90.

25. Laslett LL, Doré DA, Quinn SJ, Boon P, Ryan E, Winzenberg $\mathrm{TM}$, et al. Zoledronic acid reduces knee pain and bone marrow lesions over 1 year: a randomised controlled trial. Ann Rheum Dis. 2012;71:1322-8.

26. Varenna M, Zucchi F, Failoni S, Becciolini A, Berruto M. Intravenous neridronate in the treatment of acute painful knee osteoarthritis: a randomized controlled study. Rheumatology. 2015;54:1826-32.

27. Cassisi G, Sartori L. Efficacy of intramuscular clodronate in Complex Regional Pain Syndrome type I: description of a case located in the astragalus in a patient with psoriatic arthritis. Acta Biomed. 2009;80:268-77.

28. Varenna M, Adami S, Rossini M, Gatti D, Idolazzi L, Zucchi F, et al. Treatment of complex regional pain syndrome type I with neridronate: a randomized, double-blind, placebo-controlled study. Rheumatology (Oxford). 2013;52(3):534-42.

29. Adami S, Fossaluzza V, Gatti D, Fracassi E, Braga V. Bisphosphonate therapy of reflex sympathetic dystrophy syndrome. Ann Rheum Dis. 1997;56(3):201-4.

30. Saviola G, Abdi-Ali L, Comini L, Dalle-Carbonare LG. Use of clodronate in the management of osteoarthritis: an update. J Biol Regul Homeost Agents. 2019;33(5):1315-20.

31. Saviola G, Abdi-Ali L, Povino MR, Campostrini L, Sacco S, Dalle Carbonare L. Intramuscular clodronate in erosive osteoarthritis of the hand is effective on pain and reduces serum COMP: a 
randomized pilot trial-The ER.O.D.E. study (ERosive Osteoarthritis and Disodium-clodronate Evaluation). Clin Rheumatol. 2017;36(10):2343-350.

32. Valenti MT, Mottes M, Biotti A, Perduca M, Pisani A, Bovi M, et al. Clodronate as a therapeutic strategy against osteoarthritis. Int J Mol Sci. 2017;18(12):E2696.

33. Felson DT. The sources of pain in knee osteoarthritis. Curr Opin Rheumatol. 2005;17:624-8.

34. Link TM, Li X. Bone marrow changes in osteoarthritis. Semin Musculoskelet Radiol. 2011;15:238-46.

35. Taljanovic MS, Graham AR, Benjamin JB, Gmitro AF, Krupinski EA, Schwartz SA, et al. Bone marrow edema pattern in advanced hip osteoarthritis: quantitative assessment with magnetic resonance imaging and correlation with clinical examination, radiographic findings, and histopathology. Skelet Radiol. 2008;37:423-31.

36. Liu L, Igarashi K, Kanzaki H, Chiba M, Shinoda H, Mitani H. Clodronate inhibits PGE(2) production in compressed periodontal ligament cells. J Dent Res. 2006;85(8):757-60.

37. Chiu YG, Ritchlin DT. Denosumab: targeting the RANKL pathway to treat rheumatoid arthritis. Expert Opin Biol Ther. 2017;17(1):119-28.

38. Sims NA, Green JR, Glatt M, Schlict S, Martin TJ, Gillespie MT, et al. Targeting osteoclasts with zoledronic acid prevents bone destruction in collagen-induced arthritis. Arthritis Rheum. 2004;50(7):2338-46.

39. Oelzner P, Kunze A, Henzgen S, Thoss K, Hein G, Stein G, et al. High-dose clodronate therapy prevents joint destruction in chronic antigen-induced arthritis of the rat but inhibits bone formation at the axial skeleton. Inflamm Res. 2000;49(8):424-33.

40. Osterman T, Kippo K, Laurén L, Hannuniemi R, Sellman R. Effect of clodronate on established collagen-induced arthritis in rats. Inflamm Res. 1995;44(6):258-63.
41. Bonabello A, Galmozzi MR, Bruzzese T, Zara GP. Analgesic effect of bisphosphonates in mice. Pain. 2001;91(3):269-75.

42. Bonabello A, Galmozzi MR, Canaparo R, Serpe L, Zara GP. Long-term analgesic effect of clodronate in rodents. Bone. 2003;33(4):567-74.

43. Muratore M, Calcagnile F, Quarta E. Antalgic efficacy of the bisphosphonates in vertebral fractures of recent onset. Osteoporos Int. 2004;15(Suppl. 1):S121.

44. Rovetta G, Maggiani G, Molfetta L, Monteforte P. One-month follow-up of patients treated by intravenous clodronate for acute pain induced by osteoporotic vertebral fracture. Drugs Exp Clin Res. 2001;27(2):77-81.

45. Rovetta G, Monteforte P, Balestra V. Intravenous clodronate for acute pain induced by osteoporotic vertebral fracture. Drugs Exp Clin Res. 2000;26(1):25-30.

46. Muratore M, Canaparo R, Della Pepa C, et al. Bisphosphonates antalgic activity in recent vertebral fracture: a clodronate vs neridronate comparison. Osteoporos Int. 2004;15(Suppl. 1):S119.

47. Ernst DS, Brasher P, Hagen N, Paterson AH, MacDonald RN, Bruera E. A randomized, controlled trial of intravenous clodronate in patients with metastatic bone disease and pain. J Pain Symptom Manage. 1997;13(6):319-26.

48. Tubiana-Hulin M, Beuzeboc P, Mauriac L, Barbet N, Frenay M, Monnier A, et al. Double-blinded controlled study comparing clodronate versus placebo in patients with breast cancer bone metastases. Bull Cancer. 2001;88(7):701-7.

49. Rossini M, Viapiana O, Ramonda R, Bianchi G, Olivieri I, Lapadula G, Adami S. Intra-articular clodronate for the treatment of knee osteoarthritis: dose ranging study vs hyaluronic acid. Rheumatology (Oxford). 2009;48(7):773-8. 